\title{
Serum Albumin Levels in Severe Traumatic Brain Injury: Role as a Predictor of Outcome
}

\author{
${ }^{1}$ Department of Neurosurgery, Kasturba Medical College, Manipal \\ Academy of Higher Education (MAHE), Manipal, Karnataka, India \\ ${ }^{2}$ Department of Neurosurgery, SevenHills Hospital, Mumbai, \\ Maharashtra, India \\ ${ }^{3}$ Department of Neurosurgery, Bangur Institute of Neuroscience, \\ Kolkata, West Bengal,India \\ Indian J Neurotrauma:2020;17:24-27
}

Raghavendra Nayak ${ }^{1}$ Nitin Jagdhane ${ }^{2}$ Sanjeev Attry ${ }^{3}$ Samarendranath Ghosh ${ }^{3}$

Address for correspondence Nitin Jagdhane, MS, MCh, Department of Neurosurgery, SevenHills Hospital, Mumbai 400059, Maharashtra, India (e-mail: drjagdhane@gmail.com).

\begin{abstract}
Keywords

- traumatic brain injury

- serum albumin

- neurological outcome
\end{abstract}

Background Serum albumin has long been considered as an outcome marker in various critical illnesses. The aim of our study is to ascertain the role of serum albumin as a predictor of outcome in severe head injury patients.

Materials and Methods This is a prospective observational study of patients with severe traumatic brain injury (TBI). Depending on the serum albumin level at admission, patients were dichotomized into two groups: one with normal serum albumin and other with hypoalbuminemia. Their outcomes at 6-month follow-up were assessed by the modified Glasgow Outcome Score.

Result Eighty patients (57 males and 23 females) with severe TBI were included in the study. The mean age of the study patients was $39.6+13.1$ years and the mean serum albumin level at admission was $3.7+1.2 \mathrm{~g} / \mathrm{dL}$ with lowest being $2.2 \mathrm{mmol} / \mathrm{L}$ and highest being $6.1 \mathrm{mmol} / \mathrm{L}$. Thirty-four patients (42.5\%) had low serum albumin level $(<3.5 \mathrm{~g} / \mathrm{dL})$ at admission. At 6-month follow-up, 58 (72.5\%) patients had a good neurological outcome and $22(27.5 \%)$ had a poor outcome. The group with normal serum albumin levels showed a significantly better outcome compared with the hypoalbuminemia group $(p=0.01)$. On multiple regression analysis, low serum albumin emerged as the only predictor of the poor outcome in severe head injury patients. Conclusion Serum albumin at admission is an independent predictor of outcome in severe TBI patients. Larger prospective studies are required to confirm these findings.

\section{Introduction}

In the current era of rapid industrialization, motorization, and urbanization, traumatic brain injury (TBI) is unfolding globally as one of the leading causes of mortality and morbidity. At the global level, the estimated mortality due to TBI is $97 / 100,000$ population per year. ${ }^{1}$ It is the seventh leading cause of mortality in India accounting for $11 \%$ of total deaths (78\% of TBI are due to road traffic accidents). ${ }^{2}$ In a developing country like India, it poses a huge burden on economic status. It badly affects both patient's financial status and hospital's available resources. So, it is very important to identify the possible indicators of the outcomes of TBI which might assist to prognosticate and take appropriate decisions on the extent of treatment to be offered. ${ }^{3}$
Apart from the clinical and radiological markers, an exhaustive search is going on to identify a serum biomarker for the outcome of TBI patients. There is limited literature on the role of serum albumin in severe head injury patients although it has already been considered as a marker of outcome in other critical care settings. ${ }^{4,5}$ Serum albumin has been included in the predictive outcome models such as Acute Physiology and Chronic Health Evaluation (APACHE) III score. Considering the paucity of data over the predictive value of serum albumin at admission in head injury patients, we decided to conduct a prospective study on this. In this study, we tried to study the relationship between admission serum albumin levels and the neurological outcome in severe head injury patients. 


\section{Materials and Methods}

This was a prospective study conducted in a tertiary care hospital over a period of 18 months from 2010 to 2012. The study subjects included patients admitted with a severe head injury. The study was approved by the institutional ethics committee. Standard medical treatment and care were administered to all patients and written informed consent was obtained from each of the subject's attendant.

The inclusion criteria were:

1. Admission Glasgow Coma Score (GCS) of less than or equal to 8.

2. Age between 18 and 75 years.

3. Admission within 12 hours of trauma.

The exclusion criteria were:

1. Patients with significant multiorgan injury.

2. Pregnant women.

3. Bilateral absent pupillary light reflex, and hypotension (systolic blood pressure $<90$ ) for 10 minutes or more.

4. Patients who were lost to follow-up.

Standard care of the patients comprised of ventilation, antiseizure prophylaxis, ranitidine for gastric ulcer prophylaxis, low-molecular-weight heparin for deep vein thrombosis prophylaxis, and continuous bladder drainage. Mannitol was administered when computed tomography scan showed a focal mass effect or generalized cerebral edema. The decision of surgical intervention was individualized to each patient depending on the clinical-radiological features.

Blood sample for serum albumin was collected along with routine blood investigation within 24 hours of admission to the hospital. The serum samples were analyzed for albumin levels by bromocresol green dye binding method using an autoanalyzer XL-600 (ERBA Diagnostics Mannheim GmBH). Hypoalbuminemia was defined as a serum albumin value of less than $3.5 \mathrm{~g} / \mathrm{dL}$. Patients were divided into two groups based on the serum albumin level: one group with normal serum albumin and other with serum albumin $<3.5 \mathrm{~g} / \mathrm{dL}$ (hypoalbuminemia group).

\section{Statistical Analysis}

The data were expressed as a mean and standard deviation (SD). Chi-square test or Fisher's exact test was used to compare the proportions wherever appropriate. Multiple logistic regression analysis was conducted for adjusting well-known variables such as age, sex, and GCS. Two-sided significance tests were used throughout and the significance level was kept at $p=0.05$. Statistical analysis was done using the SPSS 11 (SPSS Inc.) software package.

\section{Ethics Statement}

The study was performed according to the Helsinki Declaration and approved by the ethical committee. Written informed consent was taken from the patient's bystanders before including the patient to the present study.
Furthermore, the present study did not interfere with the standard of care of the patients.

\section{Results}

\section{Baseline Characteristics}

Eighty patients (57 males and 23 females) with severe TBI were included in the study. The mean $( \pm$ SD) age of the study patients was $39.6+13.1$ years.

As only severe head injury patients were included in the study, GCS at admission was subdivided into two parts: better GCS (6-8/15) which included $39(48.8 \%)$ patients and poor GCS (3-5/15) which included 41 (51.2\%) patients.

The mean $( \pm \mathrm{SD})$ serum albumin level at admission was $3.7 \pm 1.2 \mathrm{~g} / \mathrm{dL}$ with lowest being $2.2 \mathrm{~g} / \mathrm{L}$ and highest being $6.1 \mathrm{~g} / \mathrm{L}$. We divided the patients into two arms depending on the serum albumin levels, the hypoalbuminemia group and normal serum albumin group. Thirty-four $(42.5 \%)$ patients had low serum albumin level $(<3.5 \mathrm{~g} / \mathrm{dL})$ at admission with remaining 46 patients (57\%) having normal serum albumin levels (-Table 1).

At 6-month follow-up, 58 (72.5\%) patients had good outcomes (modified Glasgow Outcome Scale: 4-5) and 22 had a poor outcome (modified Glasgow Outcome Scale: 1-3). Mean duration of hospital stay was $11 \pm 5$ days. The overall mortality was $22.5 \%(18 / 80)$.

\section{Chi-Square Test Analysis}

The normal serum albumin level group showed better neurological outcome compared with the hypoalbuminemia group which was statistically significant $(p=0.01)(-$ Fig. $\mathbf{1})$.

\section{Logistic Regression Analyses}

On multiple logistic regressions analysis, low serum albumin level has emerged as an independent predictor of the poor outcome in severe head injury patients ( - Table 2 ).

Table 1 General characteristics of the patients

\begin{tabular}{|l|l|}
\hline Factors & \\
\hline Age in y (mean) & $39.6+13.1$ \\
\hline Sex & $2: 1$ \\
\hline Male & $57(71 \%)$ \\
\hline Female & $23(29 \%)$ \\
\hline Serum albumin at admission (mean) & $3.7+1.2 \mathrm{~g} / \mathrm{dL}$ \\
\hline $\begin{array}{l}\text { Normal serum albumin group } \\
\text { (portion) }\end{array}$ & $46(57.5 \%)$ \\
\hline Hypoalbuminemia group (portion) & $34(42.5 \%)$ \\
\hline Glasgow Coma Scale (GCS) & \\
\hline Better GCS (6-8/15) & $39(48.8 \%)$ \\
\hline Poor GCS (3-5/15) & $41(51.2 \%)$ \\
\hline Glasgow Outcome Score (GOS) & \\
\hline Good & $58(72.5 \%)$ \\
\hline Poor & $22(27.5 \%)$ \\
\hline
\end{tabular}




\section{Discussion}

Traumatic brain injury results in the damage to the nervous system through primary and secondary insults. The primary injury directly initiates the cascade of events terminating in cell death. ${ }^{6}$ The secondary injuries to the neurons results from the series of physiological responses developed in response to the primary insults. These injuries occur secondary to the impairment of cerebral blood flow, oxygenation, metabolic functions, and autoregulations which results in the cascade of events. Inflammation plays a significant role in the secondary injury to the neuronal cells. Various inflammatory factors including cytokines, tumor necrosis factor, chemokines, and interleukins are involved in this inflammation. ${ }^{7.8}$ As serum albumin is a negative acute phase protein, its levels decrease in active inflammatory states. Hypoalbuminemia usually reflects the extent of inflammation in critically ill patients. ${ }^{9,10}$

Although there is limited literature on the association between serum albumin and TBI, there is a lot of information available for its role as a predictive factor in the critically ill patients. Considering the importance of serum albumin in various illnesses, it has been included in the predictive outcome models like the APACHE III score. ${ }^{11}$

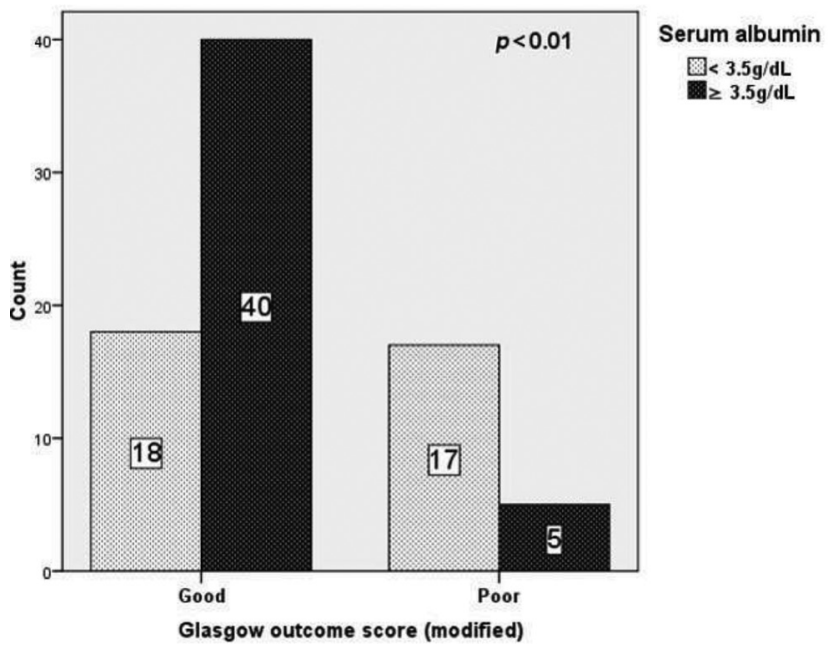

Fig. 1 Bar diagram shows that patients in the hypoalbuminemia group have got significantly poor outcome compared with the normal serum albumin level group.
Studies done by Belayev and coworkers ${ }^{12-17}$ reveal the direct influence of albumin on outcomes of neurologic injuries. Models of brain injuries (ischemic and traumatic models) show that albumin administration brings down brain infarction and edema, ${ }^{12,13,16}$ increase the cerebral perfusion, ${ }^{16}$ and decrease the histological damage to the neurons. ${ }^{14,17}$

Along with its well-known oncotic properties, albumin has also got other physiological roles including free radical scavenging, hemodilution, and decreased capillary stasis. ${ }^{18}$ Proponents of the Lund concept ${ }^{19}$ have been using the albumin to decrease edema in TBI patients and say that it is equivalent to the conventional methods given by the Brain Trauma Foundation. ${ }^{20}$

Besides its immune modulatory function, albumin also reflects the nutritional status that plays a key role in chronic debilitating conditions and critically ill patients. ${ }^{21,22}$ In acutely ill patients, hypoalbuminemia would definitely increase the chances of infections, lengthy hospital stay, and mortality. ${ }^{23}$ Because of all this, it has been an integral part of certain mathematical scores designed for the assessment of outcome in diseases involving inflammation. ${ }^{23-26}$

Multiple explanations have been given for the occurrence of hypoalbuminemia in head injury patients, which includes suppression of synthesis of albumin by the liver, increased consumption of albumin during stressful conditions, or loss of albumin during massive hemorrhage. ${ }^{27}$

Our study clearly shows a significant association between hypoalbuminemia and poor outcome of head injury patients on follow-up. Patients with hypoalbuminemia showed significantly poor neurological outcome on 6 -month follow-up $(p=0.01)$. Serum albumin emerged as an independent marker for the severity of TBI. The main limitation of our study was the small sample size and larger studies are required to further confirm these findings. Randomized control studies are required to assess the possible outcome of head injury patients on correction of hypoalbuminemia.

\section{Conclusion}

In patients with severe TBI, serum albumin is as an independent predictor of poor outcome. Larger prospective studies are required to confirm these findings.

Table 2 Multiple logistic regression analysis of factors determining the outcome of the severe head injury patients

\begin{tabular}{|l|l|l|l|}
\hline Factors & OR & $p$-Value & $95 \% \mathrm{Cl}$ \\
\hline Age $>40 \mathrm{y}$ & 0.7 & 0.18 & $0.68-7.1$ \\
\hline Male sex & 0.6 & 0.36 & $0.49-6.8$ \\
\hline Serum albumin $<3.5 \mathrm{~g} / \mathrm{dL}$ & 2.1 & 0.001 & $2.4-27.2$ \\
\hline Glasgow Coma Scale $(3-5 / 15)$ & 0.6 & 0.26 & $0.59-6.4$ \\
\hline
\end{tabular}

Abbreviations: $\mathrm{Cl}$, confidence interval; OR, odds ratio. 


\section{Conflicts of Interest}

None.

\section{References}

1 Krug EG, World Health Organization. Injury: a leading cause of the global burden of disease

2 World Health Organization, The World Health Report: Making a Difference. Geneva: World Health Organization; 1999

3 Finfer SR, Cohen J. Severe traumatic brain injury. Resuscitation 2001;48(1):77-90

4 Sung J, Bochicchio GV, Joshi M, et al. Admission serum albumin is predictive of outcome in critically ill trauma patients. Am Surg 2004;70(12):1099-1102

5 Ward RT, Colton DM, Meade PC, et al. Serum levels of calcium and albumin in survivors versus nonsurvivors after critical injury. J Crit Care 2004;19(1):54-64

6 Marik PE, Varon J, Trask T. Management of head trauma. Chest 2002;122(2):699-711

7 Corps KN, Roth TL, McGavern DB. Inflammation and neuroprotection in traumatic brain injury. JAMA Neurol 2015;72(3):355-362

8 Krug, EG \& World Health Organization. Violence and Injury Prevention Team. Injury: a leading cause of the global burden of disease. Geneva, Switzerland: World Health Organization; 1999

9 Al-Subaie N, Reynolds T, Myers A, et al. C-reactive protein as a predictor of outcome after discharge from the intensive care: a prospective observational study. Br J Anaesth 2010;105(3):318-325

10 Gabay C, Kushner I. Acute-phase proteins and other systemic responses to inflammation. N Engl J Med 1999;340(6) :448-454

11 Knaus WA, Wagner DP, Draper EA, et al. The APACHE III prognostic system. Risk prediction of hospital mortality for critically ill hospitalized adults. Chest 1991;100(6):1619-1636

12 Belayev L, Busto R, Zhao W, Clemens JA, Ginsberg MD. Effect of delayed albumin hemodilution on infarction volume and brain edema after transient middle cerebral artery occlusion in rats. J Neurosurg 1997;87(4):595-601

13 Belayev L, Zhao W, Pattany PM, et al. Diffusion-weighted magnetic resonance imaging confirms marked neuroprotective efficacy of albumin therapy in focal cerebral ischemia. Stroke 1998;29(12):2587-2599

14 Belayev L, Alonso OF, Huh PW, Zhao W, Busto R, Ginsberg MD. Posttreatment with high-dose albumin reduces histopathological damage and improves neurological deficit following fluid percussion brain injury in rats. J Neurotrauma 1999;16(6):445-453

15 Belayev L, Liu Y, Zhao W, Busto R, Ginsberg MD. Human albumin therapy of acute ischemic stroke: marked neuroprotective efficacy at moderate doses and with a broad therapeutic window. Stroke 2001;32(2):553-560

16 Ginsberg MD, Zhao W, Belayev L, et al. Diminution of metabolism/blood flow uncoupling following traumatic brain injury in rats in response to high-dose human albumin treatment. J Neurosurg 2001;94(3):499-509

17 Belayev L, Pinard E, Nallet H, et al. Albumin therapy of transient focal cerebral ischemia: in vivo analysis of dynamic microvascular responses. Stroke 2002;33(4):1077-1084

18 Dubois MJ, Vincent JL. Use of albumin in the intensive care unit. Curr Opin Crit Care 2002;8(4):299-301

19 Grände PO, Asgeirsson B, Nordström CH. Physiologic principles for volume regulation of a tissue enclosed in a rigid shell with application to the injured brain. J Trauma 1997;42(5, Suppl):S23-S31

20 Naredi S, Koskinen LO, Grände PO, et al. Treatment of traumatic head injury-U.S./European guidelines or the Lund concept. Crit Care Med 2003;31(11):2713-2714

21 Fuhrman MP, Charney P, Mueller CM. Hepatic proteins and nutrition assessment. J Am Diet Assoc 2004;104(8):1258-1264

22 McMillan DC. Systemic inflammation, nutritional status and survival in patients with cancer. Curr Opin Clin Nutr Metab Care 2009;12(3):223-226

23 Vincent JL, Dubois MJ, Navickis RJ, Wilkes MM. Hypoalbuminemia in acute illness: is there a rationale for intervention? A meta-analysis of cohort studies and controlled trials. Ann Surg 2003;237(3):319-334

24 Roxburgh CS, McMillan DC. Role of systemic inflammatory response in predicting survival in patients with primary operable cancer. Future Oncol 2010;6(1):149-163

25 Pang S, Zhou Z, Yu X, et al. The predictive value of integrated inflammation scores in the survival of patients with resected hepatocellular carcinoma: a retrospective cohort study. Int J Surg 2017;42:170-177

26 Jiang HH, Li AJ, Tang EJ, et al. Prognostic value of the combination of preoperative haemoglobin, lymphocyte, albumin, and neutrophil in patients with locally advanced colorectal cancer. Med Sci Monit 2016;22:4986-4991

27 Chen D, Bao L, Lu SQ Xu F. Serum albumin and prealbumin predict the poor outcome of traumatic brain injury. PLoS One 2014;9(3):e93167 